\title{
The DØ Detector for Run II
}

\author{
Levan Babukhadia $^{\mathrm{a} *}$ (for the $\mathrm{D} \varnothing$ Collaboration)

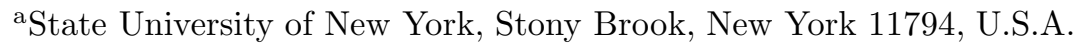

The general purpose $\mathrm{D} \varnothing$ collider detector at the Fermilab Tevatron has undergone major upgrades for Run II. We describe the current status and performance of the $\mathrm{D} \varnothing$ detector.

\section{INTRODUCTION}

The Fermilab Tevatron has undergone significant upgrades for Run II. Presently, it is delivering higher instantaneous luminosity than in Run I with a ten-fold decrease in the time between beam crossings. The Run IIa will provide an integrated luminosity of $2 \mathrm{fb}^{-1}$, about 20 times that of Run I. Also the center-of-mass energy of $p \bar{p}$ collisions has increased modestly, from 1.8 to $1.96 \mathrm{TeV}$.

To achieve the physics goals for Run II which include searches for the Higgs boson, supersymmetry, extra dimensions, and other new phenomena, as well as precision studies of weak bosons, top quark, QCD, and B-physics, the collider detectors at the Tevatron need superb electron, muon, and tau identification, excellent jet and missing transverse energy $\left(\mathscr{E}_{T}\right)$ reconstruction, and very good flavor tagging of jets through displaced vertices and leptons.

To meet these challenges in a high event rate environment, the $\mathrm{D} \varnothing$ detector has undergone major upgrades [1] (Fig. 13a). The central tracking system has been replaced by a silicon microstrip tracker, surrounded by a scintillating fiber tracker, both immersed in a $2 \mathrm{~T}$ magnetic field from a superconducting solenoid 22. To provide fast energy and position measurements for the electron trigger and offline electron identification, scintillating fiber preshower detectors have been added in the central and forward regions. Calorimeter trigger and readout electronics have been upgraded and the muon system has been significantly improved. A new forward pro-

\footnotetext{
${ }^{*}$ Current address: MS 357, Fermilab P.O. Box 500, Batavia, Illinois, U.S.A. Electronic mail: blevan@fnal.gov.
}

ton spectrometer (FPD) has been added for the diffractive and elastic physics program. To handle high event rates, an entirely new trigger and data acquisition system has been designed and implemented, see Fig. 17b.

\section{STATUS AND PERFORMANCE}

Calorimeters. The nearly compensating, uniform, and hermetic liquid-argon uranium calorimeters from Run I, with completely upgraded readout and trigger electronics, provide about 50k readout cells of electromagnetic (EM) and fine and coarse hadronic compartments with less than $0.1 \%$ dead or noisy channels. About $5 \mathrm{k}$ calorimeter towers are also used for fast trigger decisions. The system is fully commissioned.

For an in situ calibration of EM calorimeters the $Z \rightarrow e e$ signal is employed. Fig. 11c shows the $Z$ peak in a di-EM invariant mass distribution with close to expected resolution. On the other end of the spectrum, Fig. 11d shows the $J / \psi$ resonance. The calorimeter response to jets is calibrated using photon-jet data. Fig. 11e shows the distribution of $\mathbb{E}_{T}$ in multijet events, spanning several orders of magnitude; the present $\mathbb{E}_{T}$ resolution is about $7 \mathrm{GeV}$.

The Muon system consists of central and forward regions providing coverage up to $|\eta|=2$ with three layers of scintillators and drift tubes, one inside and two outside the magnetized iron toroids. In the central region, there are over $6 \mathrm{k}$ cells of proportional drift tubes and 630 (360) scintillating counters inside (outside) the toroid. In the forward regions, there are about $6 \mathrm{k} 8$ 8-cell mini drift tubes in eight octants per layer and 

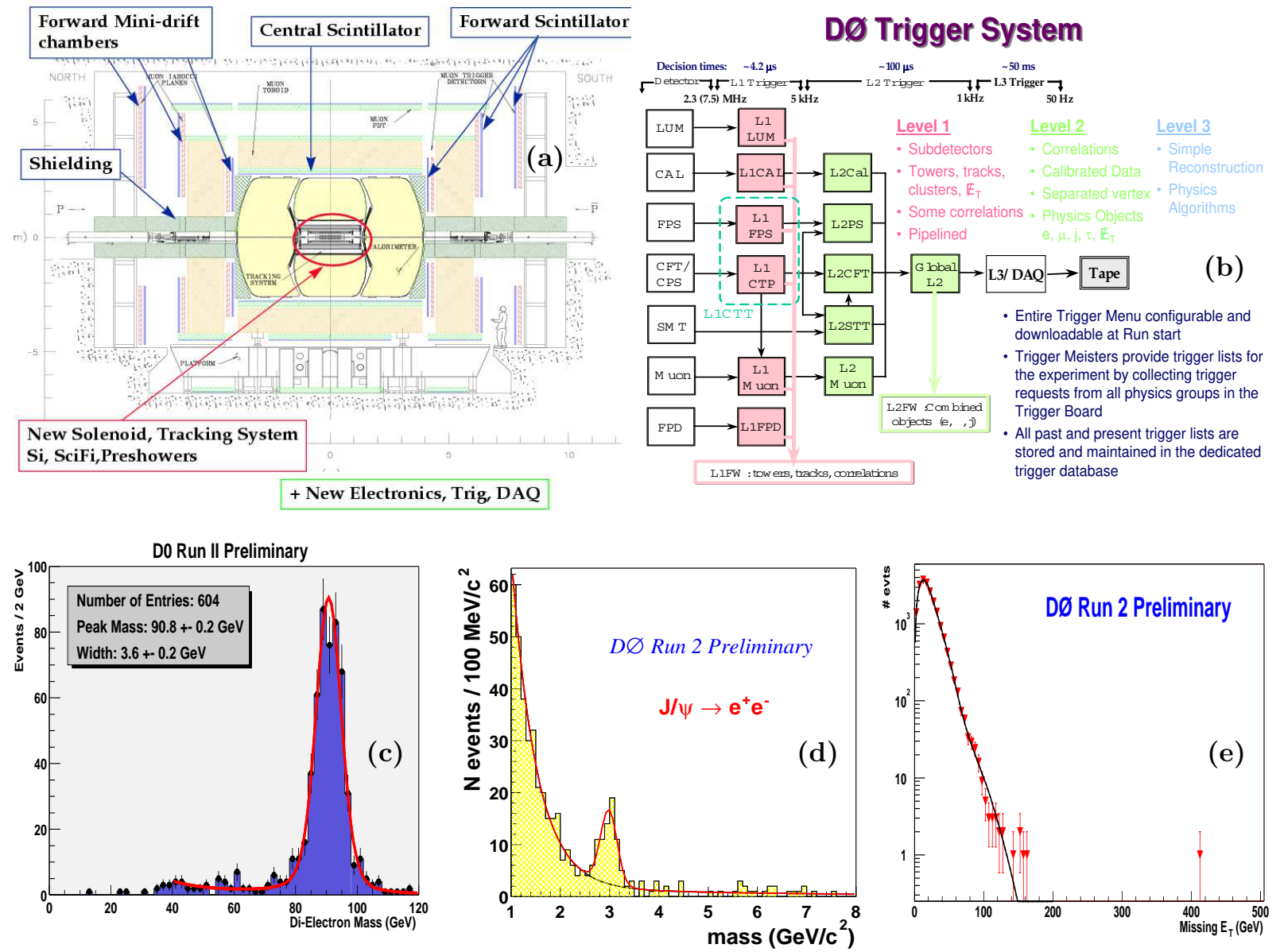

Figure 1. DØ detector for Run II (a) and diagram of the trigger system (b). Invariant mass distribution of di-EM objects showing $Z$-boson (c) and $J / \psi$ (d) resonances. Distribution of missing transverse energy $\mathbb{E}_{T}$ in multijet events (e).

over $4 \mathrm{k}$ forward scintillation counters or pixels. The muon system is fully commissioned.

Fig. 2a shows the invariant mass distribution of di-muons from the muon stand-alone system, with the $J / \psi$ resonance visible above background. Matching muons with central tracks significantly improves muon transverse momentum resolution and Figs. 2 $2 \mathrm{~b}$ and $2 \mathrm{c}$ show the $J / \psi$ and the $Z$ boson resonances, respectively. Timing cuts on muons reduce backgrounds from cosmic rays, and could aid in detection of slow moving particles.

The FPD system consists of two arms of 18 Roman pots in four quadrupole and two dipole "castles." Hits in scintillating fiber detectors installed in the Roman pots will be used for measur- ing fractional energy lost by the proton and scattering angle, and for triggering on elastic, diffractive, and double pomeron events. Currently the system is in commissioning. Several million elastic events have been recorded by FPD stand-alone system, and integration within $\mathrm{D} \varnothing$ is ongoing.

The $\mathbf{D} \varnothing$ Trigger is a three stage hardware and software system that reduces the raw event rate of about $7 \mathrm{MHz}$ down to under $50 \mathrm{~Hz}$ for recording events to tape (Fig. 1]b). The hardware triggers in Level 1 (L1) allow triggering on EM and hadronic calorimeter objects, tracks, and muons. In L2 more physics-like objects are used employing correlations and allowing triggering on separated vertices. In L3, physics algorithms are 

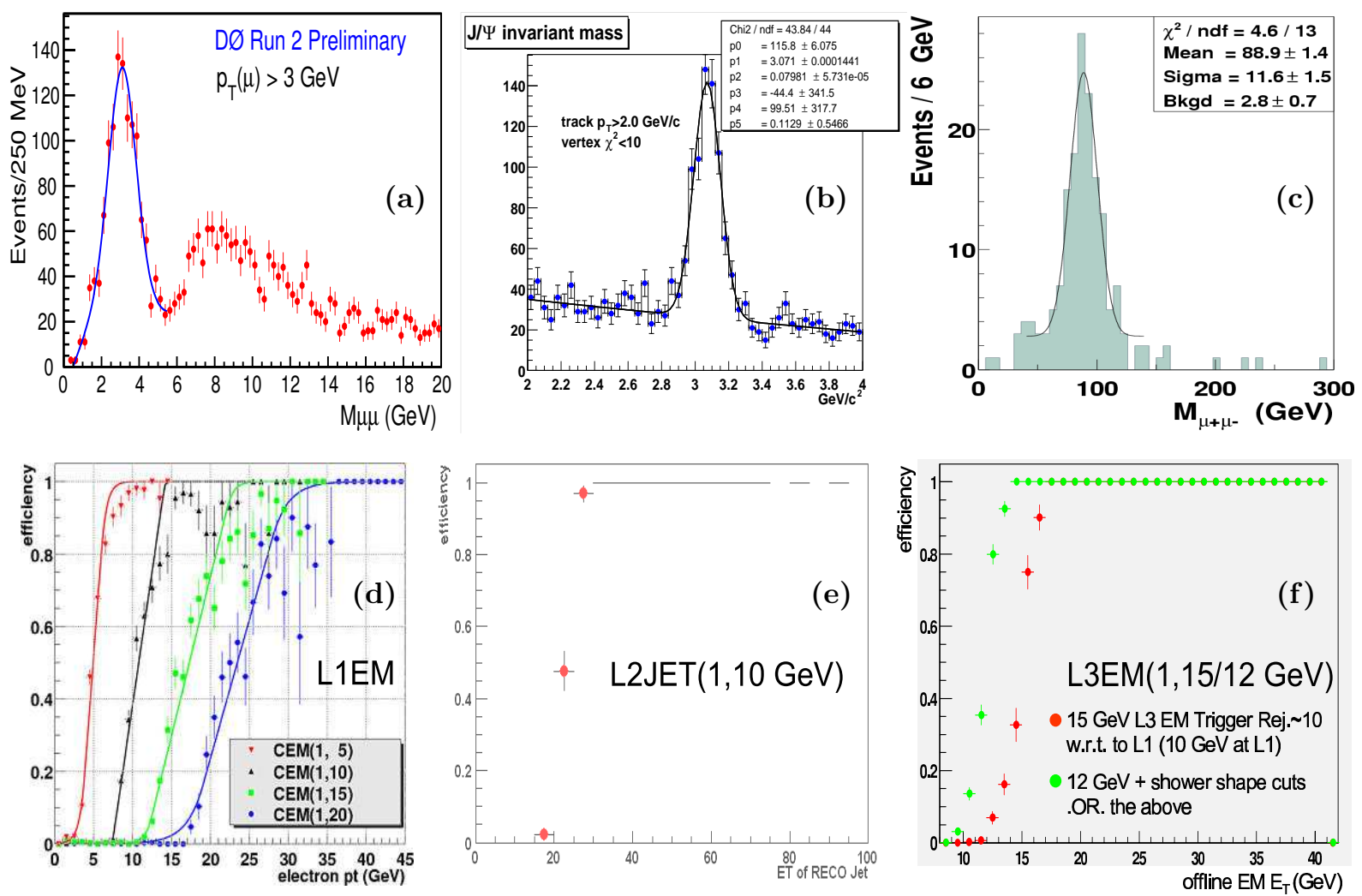

Figure 2. Invariant mass distributions of di-muons using: muon stand-alone system showing $J / \psi$ resonance (a) and muons matched with central tracks showing $J / \psi$ (b) and $Z$-boson (c) resonances. Efficiencies for triggering on electromagnetic and hadronic objects of different $E_{T}$ thresholds in the first (d), second (e), and third (f) stages of the D $\varnothing$ trigger system.

employed after fast event reconstruction.

Fig. 2d shows the efficiency "turn-on" for triggering on EM objects with various transverse energy $\left(E_{T}\right)$ thresholds using the L1 calorimeter trigger. Similarly, Fig. 2e shows the efficiency for triggering on a L2 "jet" of $E_{T}=10 \mathrm{GeV}$. Finally, Fig. 2ff shows the performance of L3 software filter to trigger on $E_{T}=15 \mathrm{GeV}$ EM objects, also indicating that the $E_{T}$ threshold can effectively be lowered by utilizing EM shower shape requirements.

\section{SUMMARY AND OUTLOOK}

The D $\varnothing$ detector for Run II is operating and collecting physics data. There has been enormous progress over the past year in installation, integration, and commissioning of the de- tector, and understanding the data. Performance of the Run II DØ detector is very encouraging, all subdetectors are operating well, software and computing systems are working well, we are reconstructing electrons, muons, jets, $\mathbb{E}_{T}, J / \psi$ 's, $W$ 's and Z's. We are working hard to complete commissioning, improve calibration and alignment, optimize detector, trigger, and DAQ performance, and are on the way to exciting physics, with first physics results now appearing [3].

\section{REFERENCES}

1. The DØ Experiment, www-d0.fnal.gov.

2. G. Ginther, these proceedings.

3. M. Narain, these proceedings. 